\title{
O PERFIL DE DOCENTES PARA O ENSINO TUTORIAL DE CURSOS DE MEDICINA: UM PROCESSO ORIENTADOR PARA A CONSTRUÇÃO DE PROBLEMAS DA REALIDADE LOCAL
}

\author{
PERFIL DE PROFESORES PARA LA ENSEÑANZA TUTORIAL DE CURSOS DE \\ MEDICINA: UN PROCESO GUÍA PARA LA CONSTRUCCIÓN DE PROBLEMAS DE \\ REALIDAD LOCAL
}

PROFILE OF TEACHERS FOR THE TUTORIAL TEACHING OF MEDICINE COURSES: A GUIDING PROCESS FOR THE CONSTRUCTION OF LOCAL REALITY PROBLEMS

RESUMO: Os cursos de Medicina possuem em suas diretrizes curriculares a proposta de implementar um ensino baseado na comunidade em que a instituição se encontra inserida. Objetiva-se conhecer o perfil dos docentes do Ensino Tutorial de um curso da Medicina. Tratase de um estudo descritivo, quantitativo, prospectivo, realizado com os docentes do módulo de ensino tutorial nos semestres 2018.2 e 2019.1 do curso de Medicina de uma instituição pública, sendo que $50 \%$ dos docentes estão entre 5 e 10 anos de carreira docente. Todos encontravamse com experiência no ensino tutorial. A maioria (57\%) encontrava-se há pelo menos 2 anos sem capacitação voltada para ensino tutorial. Os docentes participantes eram dos mais diversos cursos. A maioria (57\%) respondeu estarem próximos ou muito próximos da realidade local antes do ensino tutorial. Mesmo após sua inserção no ensino tutorial, $43 \%$ dos docentes relataram pouca proximidade. Todos os docentes responderam que possuíam conhecimento razoável sobre os temas discutidos na tutoria. 29\% consideraram-se pouco próximos da etapa de intervenção realizada pelos discentes no ensino tutorial. $83 \%$ relataram que consideravam razoável o aprendizado sobre os temas discutidos. Foram abordados os mais variados temas no ensino tutorial: doenças transmissíveis e não transmissíveis, doenças parasitárias, e problemas ambientais e sociais.

PALAVRAS-CHAVE: Docentes de medicina. Educação médica. Tutoria.

RESUMEN: Los cursos de medicina tienen en sus pautas curriculares la propuesta de implementar la enseñanza basada en la comunidad en la que se inserta la institución. El objetivo es conocer el perfil de los docentes de Educación Tutorial en un curso de medicina. Este es un estudio descriptivo, cuantitativo y prospectivo, realizado con los maestros del módulo de enseñanza tutorial en los semestres 2018.2 y 2019.1 del curso de medicina de una institución pública. El 50\% de los docentes tienen entre 5 y 10 años de carrera docente. Todos tenían experiencia con la enseñanza tutorial. La mayoría (57\%) había estado sin capacitación durante al menos 2 años para la enseñanza tutorial. Los profesores participantes pertenecían a los cursos más diversos. La mayoría (57\%) respondió que están cerca o muy cerca de la

${ }^{1}$ Universidade Federal do Maranhão (UFMA), Pinheiro - MA - Brasil. Professora Adjunta do Curso de Medicina. Doutorado em Saúde Coletiva (UFMA). ORCID: https://orcid.org/0000-0001-7193-4861. E-mail: amanda_namibia@hotmail.com

Temas em Educ. e Saúde, Araraquara, v. 16, n. 1, p. 133-141, jan./jun., 2020. e-ISSN 2526-3471. 
realidad local antes de la enseñanza tutorial. Incluso después de su inserción en la enseñanza tutorial, el $43 \%$ de los maestros que informaron poca proximidad. Todos los maestros respondieron que tenían un conocimiento razonable sobre los temas discutidos en la tutoría. El 29\% se consideraba no cercano a la etapa de intervención realizada por los estudiantes en la enseñanza tutorial. $83 \%$ de los professores informaron que consideraban que aprender sobre los temas discutidos era razonable. Los temas más variados fueron cubiertos en la enseñanza tutorial: enfermedades transmisibles y no transmisibles, enfermedades parasitarias $y$ problemas ambientales y sociales.

PALABRAS CLAVE: Docentes médicos. Educación médica. Tutoría.

ABSTRACT: Medicine courses have in their curricular guidelines a proposal for the implementation of teaching based on the community that the institution is inserted. The objective here is to know the profile of the teachers of the Tutorial Teaching of a medical course. This is a descriptive, quantitative, prospective study, carried out with the professor of the tutorial teaching module in the 2018.2 and 2019.1 semesters of the medical course of a public institution, with $50 \%$ of the teachers having between 5 and 10 years of teaching career. All were experienced with tutorial teaching. The majority (57\%) found themselves for at least 2 years without capacitation aimed at tutorial teaching. The participating teachers were from the most diverse courses. The majority (57\%) said they were close or very close to the local reality before tutorial teaching. Even after their insertion in tutorial teaching, $43 \%$ of the teachers reported little proximity. All teachers responded that they had reasonable knowledge about the topics discussed in tutoring. 29\% considered little to follow the intervention step performed by students in tutorial teaching. $83 \%$ reported that learning about the topics discussed is considered reasonable. The most varied topics were covered in tutorial teaching: communicable and non-communicable diseases, parasitic diseases and, environmental and social problems.

KEYWORDS: Medical teachers. Medical education. Mentoring.

\section{Introdução}

As diretrizes curriculares nacionais (DCN), para os cursos de graduação, foram construídas como estratégia para direcionar como deve ser construído e implementado o projeto político pedagógico (PPP) de uma instituição de ensino superior (IES). No curso de Medicina, avanços têm ocorrido com a construção dos PPP baseados nas atuais diretrizes, e cada vez mais os discentes estão inseridos nos serviços de saúde e na comunidade.

Para as DCN, o Curso de Graduação em Medicina deve ter um projeto político pedagógico, construído coletivamente, de forma que o aluno seja o centro do processo de ensino aprendizagem. Nessa proposta, o docente desempenha o papel de facilitador desse processo, com estratégias mediadoras que irão apoiar o discente na construção de seu aprendizado (BRASIL, 2001a). 
Entende-se então que as DCNs buscam contribuir para a inovação e a qualidade do PPP dos cursos de Graduação em Medicina, de forma que haja a orientação para um currículo que se atente para o perfil acadêmico e profissional do egresso. Discute-se ainda que aspectos como compreensão, interpretação, preservação, reforço, fomento e difusão das culturas e histórias internacionais, nacionais e regionais de cada localidade, devem ser considerados, com a compreensão do pluralismo e diversidade cultural que vivenciamos no Brasil (BRASIL, 2001b).

Há o desafio evidenciado para uma reformulação do ensino de saúde, tanto com relação aos seus objetivos quanto às suas práticas. Os cursos de Medicina buscam nesse momento, tentar se adequar às novas reivindicações das DCNs, tanto do ponto de vista ético quanto do social (AYRES, 2013).

O Ministério da Educação, em 2014, estabeleceu nas DCNs, que o curso de Medicina possui responsabilidade social e compromisso com a defesa da cidadania, dignidade humana e saúde integral da população (BRASIL, 2014).

Uma estratégia para se alcançar essa proposta são os PPPs articulados no tripé de sustentação da instituição superior de ensino: ensino, pesquisa e extensão, com o intuito de proporcionar uma formação integral. Dessa forma, com a proposta de fortalecer esse tripé e tentar fomentar no aluno a sua inserção na comunidade e serviços de saúde, as DCNs propuseram inserir na grade curricular atividades de tutoria, esta denominada nos currículos acadêmicos da Universidade Federal do Maranhão (UFMA) como Eixo Integrador (BRASIL, 2001a).

Em 2014, as DCNs, para a Graduação de Medicina, reforçaram a necessidade do curso possuir sua formação centrada na Atenção Básica, de forma que a assistência ocorra na rede de atenção à saúde no Sistema Único de Saúde (SUS), iniciando no serviço de saúde que deve ser a porta de entrada do usuário. Ao se trabalhar com a proposta do Eixo Integrador, mostra-se aqui, então, a formação médica, a atenção a saúde prestada, e a participação popular, os quais são aspectos indissociáveis ao se buscar a integração ensino-serviço-comunidade (BRASIL, 2014).

O Eixo Integrador trabalha nesse foco devido a ideia de se fortalecer o movimento sanitarista que, com a criação do SUS, discute as limitações de uma formação tradicional e reforça a necessidade de um modelo educacional que priorize uma formação médica generalista, humanista e crítico-reflexiva. Esse novo modelo de saúde tem como aposta um profissional que atente não apenas para o processo saúde-doença com uma abordagem 
tecnicista, mas que possa ir além disso: que busque dimensões políticas e éticas que se encontram na prática cotidiana (AYRES, 2013).

[...] como resposta a uma política interministerial, configurada como agenda da Reforma Sanitária Brasileira, no que se refere ao ordenamento da formação na área da saúde, aproximando as práticas de ensino, pesquisa e extensão ao sistema de saúde e às demandas sociais (ANDRADE; SILVA; SILVA, 2011, p. 57).

Além da busca por um currículo focado na atenção básica, as DCNs propuseram o uso de metodologias ativas nas avaliações institucionais, de forma a propiciar aos discentes e docentes múltiplas ideias e olhares, envolvendo todos os atores presemtees no processo. A proposta dessas metodologias é que haja a compreensão dos conceitos considerados chave para o aprendizado, para um desenvolvimento de um pensamento crítico, para a aplicação prática do contexto teórico, e para um bom desempenho individual e coletivo (BRASIL, 2014).

Apesar das propostas evidenciadas nas DCNs, ainda se observa que o modelo adotado no Eixo Integrador nos cursos de Graduação em Medicina encontra-se ineficiente, sendo importante uma ênfase na abordagem pedagógica que tem como objetivo a humanização da formação médica. Isso é possível com o estreitamento na interação do aluno com a comunidade e a realidade vivenciada pelos seus clientes, o que vem a futuramente facilitar a relação médicopaciente e, como consequência, o processo diagnóstico-terapêutico (AYRES, 2013).

Refletir sobre a formação dos estudantes de Medicina, demonstra a preocupação em como será futuramente a sua atuação enquanto médico. Dessa forma, a ideia de se trabalhar com o ensino tutorial não deve limitar-se a problemas incomuns com a realidade daquela comunidade, mas sim deve considerar o pressuposto de que uma discussão de qualidade é aquela em que o aluno pode colocar em prática em seu ambiente de extensão acadêmica. Devese então privilegiar as competências profissionais construídas, e ter por base uma prática reflexiva, que é pautada pelo envolvimento profissional ético (FONSECA; IDE; PETERLINE, 2013).

A aprendizagem deve ser interpretada como um caminho que possibilita ao sujeito social transformar-se e transformar seu contexto. “[...] deverá ter a investigação como eixo integrador que retroalimenta a formação acadêmica e a prática do médico" (BRASIL, 2001b, p. 1).

Diante do que já foi exposto, definiu-se como problema de pesquisa: Como reorientar a construção de problemas para o ensino tutorial a partir da realidade local em um curso de Medicina? Este estudo torna-se relevante ao se compreender a necessidade de haver uma 
orientação e acompanhamento das atividades do ensino tutorial em todos os períodos acadêmicos do curso de Graduação de Medicina.

É importante construir os casos explorando a realidade da comunidade e dos serviços de saúde local, de forma a buscar soluções teóricas para os problemas locais. Desde seu momento em sala de aula, é relevante inserir os acadêmicos do curso de Medicina, no seu local de atuação.

Dessa forma, propôs-se inicialmente conhecer os docentes inseridos no Ensino Tutorial de um curso da Medicina.

\section{Métodos}

\section{Tipo de estudo}

Trata-se de um estudo do tipo descritivo, com abordagem quantitativa, prospectivo.

\section{População e período do estudo}

Foram participantes da pesquisa os docentes dos módulos de ensino tutorial, do curso de Medicina. A pesquisa ocorreu no período de janeiro a julho de 2019.

\section{Coleta dos dados}

Inicialmente foi realizado um convite a todos os docentes do ensino tutorial. Logo após, foi entregue o termo de consentimento livre e esclarecido (TCLE) para cada participante, garantindo o anonimato dos participantes, assim como o direito de desistirem de participar do estudo a qualquer momento.

Foram coletados dados sobre o perfil docente, a experiência dos docentes. A coleta se deu por meio de um questionário individual elaborado no programa Survey Monkey ${ }^{2}$, que foi encaminhado por e-mail, com a proposta de conhecer o perfil docente.

\section{Análise dos dados}

Os dados qualitativos foram analisados no programa Survey Monkey. Foi realizada análise descritiva por meio de frequência absoluta e relativa, média e desvio padrão.

\section{Aspectos éticos}

${ }^{2}$ Programa disponível em: www.surveymonkey.com

Temas em Educ. e Saúde, Araraquara, v. 16, n. 1, p. 133-141, jan./jun., 2020. e-ISSN 2526-3471. 
Para atender aos critérios éticos, foram seguidas as recomendações determinadas na Resolução $n^{\circ}$ 466/12 do Conselho Nacional de Saúde (BRASIL, 2012). O projeto foi encaminhado à Secretaria Municipal de Saúde, para autorização da pesquisa nas unidades de saúde. O projeto também foi submetido à Plataforma Brasil, para apreciação e recebimento de parecer favorável de número 102204/2017. Àqueles que desejaram participar do estudo foi solicitado que assinassem o Termo de Consentimento Livre e Esclarecido em duas vias, nas quais os objetivos e finalidades da investigação foram esclarecidos, e assegurado o anonimato dos participantes e o consentimento para a divulgação dos resultados obtidos.

\section{Resultados e discussão}

O ensino tutorial neste curso da Medicina é formado por 13 docentes. Destes 50\% estão entre 5 e 10 anos de carreira docente, $34 \%$ estão há menos de 5 anos e $16 \%$ há mais de 10 anos na atividade da docência. Todos encontravam-se com experiência no ensino tutorial, estando há pelo menos 3 semestres atuando nessa forma de ensino. A maioria (57\%) encontrava-se há pelo menos 2 anos sem capacitação voltada para ensino tutorial. Os docentes participantes eram dos mais diversos cursos: médicos, enfermeiros, farmacêuticos, odontólogos, nutricionista e fisioterapeuta.

Quando perguntados sobre a aproximação com o serviço de saúde do município, a maioria (57\%) respondeu estarem próximos ou muito próximos da realidade local. No entanto, quando questionado sobre a proximidade após sua inserção no ensino tutorial, o percentual não alterou, mostrando que os $43 \%$ dos docentes que relataram pouca proximidade, permaneceram desconhecendo a realidade local.

Todos os docentes responderam que possuíam conhecimento razoável sobre os temas discutidos no ensino tutorial. Destaca-se que $29 \%$ dos docentes consideraram-se pouco próximos da etapa de intervenção realizada pelos discentes no ensino tutorial.

Quando questionados sobre sua percepção sobre o conhecimento discente, $83 \%$ relataram que consideravam razoável o aprendizado sobre os temas discutidos. Quando questionados sobre quais eram esses temas, percebeu-se que são abordados os mais variados temas: doenças transmissíveis e não-transmissíveis, doenças parasitárias, e problemas ambientais e sociais.

A proposta de se trabalhar a tutoria, trouxe ao curso de Graduação de Medicina novas perspectivas de discussões críticas, no entanto, até o momento, limitada a se trabalhar apenas 
para o crescimento intelectual do estudante. Essa problemática traz ao aluno um distanciamento da realidade dos serviços de saúde e da comunidade em que está inserido.

Os docentes do curso devem, então, atentar-se para as especificidades do local, no que se refere às suas características sócio-sanitárias. É de conhecimento tanto discente quanto docente de que os currículos devem ser baseados na comunidade, o que ainda é uma realidade distante dos casos trabalhados no ensino tutorial.

Haddad (2009) explica a importância de se buscar no ensino tutorial a integração do ensino com a rede de atenção a saúde do SUS instituído como ato pedagógico, esta viabilizada através de um alinhamento e organização da política de educação na saúde.

Para se viabilizar a construção do ensino tutorial baseado nos serviços de saúde e na comunidade, é importante antes de tudo conhecê-los. Nesse processo, a participação ativa de representantes profissionais de saúde da rede de serviços de saúde e usuários do SUS é imprescindível.

Busca-se aqui, uma aproximação dos profissionais das práticas pedagógicas além de proporcionar aos docentes do curso de Medicina sua inserção e conhecimento dos processos de atenção em saúde local. Isto possibilita a continuidade e a transformação constante de ambos os processos, quer seja no ensino, quer seja na prestação de serviços de saúde (HADDAD, 2009).

Para Bollela et al., (2014) o ato de educar para os cursos de graduação em saúde deve ter como meta a preparação de seus estudantes de forma que desenvolvam competências e habilidades para uma prática profissional responsável, ou seja, profissionais a serviço da comunidade.

Compreende-se a importância de se ofertar o ensino tutorial aos acadêmicos de Medicina, visando a obtenção do domínio teórico sobre os diversos assuntos que envolveram suas atividades cotidianas nos serviços de saúde. No entanto, suas ações e atividades executadas após a formação não devem estar focadas apenas no que sabem, mas no que fazem com o conhecimento ou a partir dele (BOLLELA et al., 2014).

Além disso, as discussões baseadas na realidade dos serviços de saúde e na comunidade proporcionam uma grande oportunidade de se descobrir lacunas, as quais podem ser pesquisadas através de propostas de projetos de pesquisa e ações de maior impacto social e de saúde podem ser alcançadas com projetos de extensão. 


\section{Considerações finais}

Incentivar o fortalecimento do ensino, pesquisa e extensão universitária através da realidade local proporciona, além de tudo, a educação permanente em saúde, de forma que as discussões irão além dos muros da universidade, podendo perpassar todos os locais de prática dos acadêmicos de Medicina. O conhecimento nesse momento, não é adquirido apenas pelos discentes, mas alcança os docentes da universidade e profissionais de saúde que atendem a comunidade.

Percebe-se que existe, então, o desafio de sensibilizar os docentes e discentes do curso de Graduação em Medicina de forma que o ensino tutorial deva fomentar a construção de discussões baseadas na realidade da comunidade atendida e nos serviços de saúde local.

Deve-se haver uma reflexão de que, desde a graduação, os estudantes devem estar inseridos no espaço em que se encontram profissionalmente, reconhecendo as necessidades e peculiaridades da população local. E, então, a partir desse reconhecimento, o processo de prestação de serviços de saúde será direcionado, de forma a atender as especificidades locais, para qualquer local em que os acadêmicos se encontrem, por entenderem que as ações devem ser prestadas de acordo com a realidade vivenciada.

\section{REFERÊNCIAS}

ANDRADE, M. R. S.; SILVA, C. R. L. D.; SILVA, A. Formação em saúde: experiências e pesquisas nos cenários de prática, orientação teórica e pedagógica. Edifurb, 2011.

AYRES, J. R. C. M. et al. Humanidades como disciplina da graduação em Medicina. Rev. bras. educ. med., Rio de Janeiro, v. 37, n. 3, p. 455-463, set. 2013. Disponível em: http://www.scielo.br/scielo.php?script=sci_arttext\&pid=S010055022013000300019\&lng=en\&nrm=iso. Acesso em: 14 out 2019.

BOLLELA, V. R. et al. Educação baseada na comunidade para as profissões da saúde: aprendendo com a experiência brasileira. Ribeirão Preto, SP: FUNPEC-Editora, 2014.

BRASIL. Ministério da Educação (MEC). Diretrizes Curriculares Nacionais dos Cursos de Graduação em Enfermagem, Medicina e Nutrição. Diário oficial da União, Brasília, DF, 03 out. 2001a. Disponível em: http://portal.mec.gov.br/dmdocuments/ces1133.pdf. Acesso em: 14 out 2019.

BRASIL. Diretrizes Curriculares para o Curso de Medicina. Diário oficial da União, Brasília, DF, 09 nov. 2001b. Disponível em:

http://portal.mec.gov.br/cne/arquivos/pdf/CES04.pdf. Acesso em: 14 out 2019.

Temas em Educ. e Saúde, Araraquara, v. 16, n. 1, p. 133-141, jan./jun., 2020. e-ISSN 2526-3471. 
BRASIL. Ministério da Educação (MEC). Diretrizes Curriculares Nacionais do curso de graduação em Medicina. Resolução $n^{\circ}$ 3, de 20 de junho de 2014. Diário oficial da União, Brasília, DF, 23 jun. 2014. Disponível em:

http://portal.mec.gov.br/index.php?option=com_docman\&view=download\&alias=15874rces003-14\&category_slug=junho-2014-pdf\&Itemid=30192. Acesso em: 14 out 2019.

FONSECA, A. S.; IDE, C. C.; PETERLINE, F. L. Delineamento para a construção de um projeto pedagógico do curso de Medicina: relato de experiência. O Mundo da Saúde, São Paulo, v. 37, n. 1, p. 84-88, 2013. Disponível em: http://www.saocamilosp.br/pdf/mundo_saude/101/8.pdf. Acesso em: 14 out 2019.

HADDAD, A. E. O Departamento de Gestão da Educação na Saúde frente aos desafios para a consolidação do Sistema Único de Saúde. In: AMÂNCIA, F. O.; PACHECO, S. P. Mestrado profissional em gestão do trabalho e da educação na saúde: ação e reflexões. Rio de Janeiro: Ed. Fiocruz; p. 15-19, 2009.

PEREIRA, O. P.; ALMEIDA, T. M. C. Medical education according to a resistance pedagogy. Interface - Comunic., Saúde, Educ., v. 9, n. 16, p. 69-79, 2005.

\section{Como referenciar este artigo}

PASKLAN, Amanda Namíbia Pereira. O perfil de docentes para o ensino tutorial de cursos de medicina: um processo orientador para a construção de problemas da realidade local. Temas em Educ. e Saúde, Araraquara, v. 16, n. 1, p. 133-141, jan./jun., 2020. e-ISSN 2526-3471. DOI: https://doi.org/10.26673/tes.v16i1.13591

Submetido em: 17/02/2020

Revisões requeridas: 20/04/2020

Aprovado em: 30/05/2020

Publicado em: 19/06/2020 